\title{
Time-Dependent Strength of Colloidal Gels
}

\author{
S. Manley, ${ }^{1}$ Benny Davidovitch, ${ }^{2}$ Neil R. Davies, ${ }^{1}$ L. Cipelletti, ${ }^{1, *}$ A. E. Bailey,,${ }^{1, \dagger}$ R. J. Christianson, ${ }^{1}$ U. Gasser, ${ }^{1, \ddagger}$ \\ V. Prasad, ${ }^{1,8}$ P. N. Segre, ${ }^{1, \|}$ M. P. Doherty, ${ }^{3}$ S. Sankaran, ${ }^{3}$ A. L. Jankovsky, ${ }^{3}$ B. Shiley, ${ }^{4}$ J. Bowen, ${ }^{4}$ J. Eggers, ${ }^{4}$ C. Kurta, ${ }^{4}$ \\ T. Lorik, ${ }^{4}$ and D. A. Weitz ${ }^{1}$ \\ ${ }^{1}$ Department of Physics \& DEAS, Harvard University, Cambridge, Massachusetts 02138, USA \\ ${ }^{2}$ DEAS, Harvard University, Cambridge, Massachusetts 022138, USA \\ ${ }^{3}$ NASA Glenn Research Center, Brook Park, Ohio 44135, USA \\ ${ }^{4}$ Zin Technologies, Inc., Brook Park, Ohio 44142, USA \\ (Received 27 July 2004; published 22 July 2005)
}

\begin{abstract}
Colloidal silica gels are shown to stiffen with time, as demonstrated by both dynamic light scattering and bulk rheological measurements. Their elastic moduli increase as a power law with time, independent of particle volume fraction; however, static light scattering indicates that there are no large-scale structural changes. We propose that increases in local elasticity arising from bonding between neighboring colloidal particles can account for the strengthening of the network, while preserving network structure.
\end{abstract}

Gels are dilute connected networks which are capable of supporting applied stresses; they are commonly used to control the rheological properties of complex materials. Such networks can be formed by the aggregation of colloidal particles, which occurs when an attractive interaction is induced between them. Network elasticity is highly sensitive to the connectivity and arrangement of particles in the constituent aggregates. Colloidal gels are out-ofequilibrium systems; as a result, these networks frequently display time-dependent properties, due to network restructuring. This kind of aging is modeled for generic nonequilibrium systems as an evolution toward lower energy states, as the system explores a complex energy landscape [1,2]. However, network elasticity is also dependent on the interactions between particles; therefore, time-dependent interactions may also lead to changes in network properties. For colloidal gels, it is generally assumed that interparticle attractions, such as those described by Derujaguin-LandauVerwey-Overbeek [3,4] or Asakura-Oosawa potentials [5], determine local bond elasticity, thus, in principle, limiting their strength [6,7]. In the absence of a steric stabilization layer, particle interactions can also arise from physical bonds, as a result of covalent bonding or polymer entanglements. Within this framework, time evolution of network properties can be linked to the interparticle potential, for example, through the transport of particles from secondary to primary minima [8]. Aging can also be a consequence of time-dependent physical bonding; for example, the sintering of aggregated polystyrene particles is thought to drive local shrinkage that deforms the network [9]. The strength of the local interparticle bonds will have a direct impact on the network elasticity; however, these critical effects have never been explored.

In this Letter, we present measurements of the elasticity of colloidal silica gels. Surprisingly, we find that, upon gelation, their storage moduli $G^{\prime}$ increase as a power law in time, $G^{\prime} \sim t^{0.4}$, independent of initial volume fraction $\phi$. Moreover, the time evolution of the network persists long after gelation occurs, for the duration of the measurement. As a consequence, their elasticity maintains the same volume fraction dependence, $G^{\prime} \sim \phi^{3.6}$, independent of time. We postulate that the stiffening of the network is a result of chemical reactions at the junctions between neighboring particles, which increase local bond strength. We introduce a simple model based on increasing local bond area to account for the observed data.

We use charge-stabilized silica colloids with a radius $a=10 \mathrm{~nm}$ in water at $25^{\circ} \mathrm{C}$; we initiate aggregation by adding $\mathrm{MgCl}_{2}$ to a concentration of $40 \mathrm{mM}$. Gravitational loading may induce network restructuring since $\Delta \rho=$ $1.27 \mathrm{~g} / \mathrm{cm}^{3}$. To circumvent this, we perform experiments in microgravity. We study a gel at $\phi=2 \times 10^{-4}$; this is in the range of concentration where aging is observed in polystyrene gels [9]. For experiments in microgravity, we use the Physics of Colloids in Space apparatus on the International Space Station (ISS) [10,11]. To form the sample in space, the salt and colloid solutions are stored in separate chambers, sealed with a pinch valve. When the valve is released, two metal rods push in an alternating fashion on the rubber fluid bladders backing the chambers to mix the solutions.

To obtain information about the structure and elasticity of colloidal gels at low $\phi$, we perform both static light scattering (SLS) and dynamic light scattering (DLS) over a wide range of angles [12]. The aggregation process results in a relatively monodisperse size distribution of clusters, which is reflected by a peak in the static scattering intensity $I(q)$; this peak shifts to a lower wave vector $q$ and increases in intensity as clusters grow [13]. Fractal clusters are formed, as evidenced by the power law decay of $I(q)$; we show in Fig. 1 (inset) data obtained on the ISS, for different times after aggregation was initiated. The fractal dimen- 


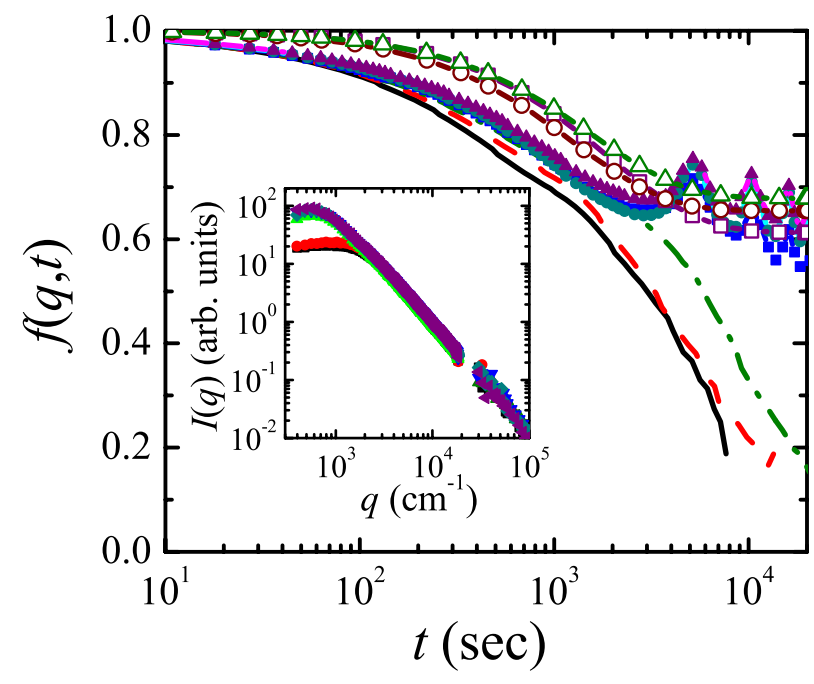

FIG. 1 (color online). Dynamic structure factors at $q=$ $3771 \mathrm{~cm}^{-1}$; raw data at 12 (solid line), 18 (dashed line), 40 (dash-dotted line), $55(\boldsymbol{\square}), 69(\mathbf{O})$, and $93 \mathrm{~h}(\mathbf{\Delta})$ since initialization and corrected for external temperature fluctuations at $55(\square), 69(\bigcirc)$, and $93 \mathrm{~h}(\triangle)$. Inset: Static light scattering between 1 and $65 \mathrm{~h}$ after initialization. No changes are observed after $\sim 15 \mathrm{~h}$.

sion is $d_{f}=1.9$, consistent with aggregation that may begin slightly in the reaction-limited regime, but crosses rapidly into diffusion-limited cluster aggregation (DLCA) $[14,15]$. Roughly $12 \mathrm{~h}$ after initialization, the SLS stops evolving in time. This is consistent with the onset of gelation, which occurs when the colloidal aggregates grow large enough to form a space-filling network. For DLCA gels, the average cluster size at gelation is $R_{c}=$ $a \phi_{0}^{1 /\left(d_{f}-3\right)}$ [16]. We calculate $R_{c}=2.3 \times 10^{-3} \mathrm{~cm}$ for our sample; this corresponds to a wave vector $q=1360 \mathrm{~cm}^{-1}$, and is in good agreement with the final position of the peak of $I(q)$.

Gels at such low $\phi$ are extremely weak, and cannot support the stresses necessary for typical bulk rheological measurements. Instead, we measure the dynamic structure factor, $f(q, \tau)$, which provides information about their structural relaxation, and hence their elasticity. Even after SLS suggests that the sample is a gel, $f(q, \tau)$ still shows a complete relaxation at $q=3771 \mathrm{~cm}^{-1}$, as shown by the data plotted as lines in Fig. 1. As the sample ages, its relaxation time increases, as seen for $t=40 \mathrm{~h}$ after initialization. At even longer times, the decay becomes arrested. The resultant dynamic structure factor is approximated by $f_{g}(q, \tau)=\exp \left(-q^{2}\left\langle\Delta r^{2}\right\rangle / 6\right)$, where the mean squared displacement of a segment of the gel, $\left\langle\Delta r^{2}\right\rangle=\delta^{2}\left(1-\exp \left[-\left(\tau / \tau_{0}\right)^{p}\right]\right)$, results from its overdamped modes, with $\tau_{0}$ the viscous damping time, and $\delta^{2}$ the maximum deviation [17]. At long delay times, particle motions are constrained by the network; thus, the dynamics saturate to a plateau when the maximum mean squared displacement is less than $1 / q$. In these experiments performed on the ISS, an unusual oscillation is seen on top of the developing plateau. Its period is independent of $q$, and corresponds to the $83 \mathrm{~min}$ orbital period of the ISS; temperature sensors located near the apparatus record an oscillation of the same period. We therefore correct the correlation functions by fitting to a convolution of $f_{g}(q, \tau)$ with an oscillatory function with a period of $83 \mathrm{~min}$. The fit parameters allow us to determine the unperturbed $f_{g}(q, \tau)$, shown in Fig. 1 as open symbols. The plateau height $f_{g}(q, \tau=\infty)$ increases with sample age; thus, the particle displacements become increasingly constrained over time, consistent with an increase in gel elasticity. By contrast, SLS shows that no significant changes in the structure of the gel occur over the same time period.

To determine the generality of this aging behavior, we investigate the evolution of the network at higher $\phi$; this has the advantage that the elastic moduli are large enough to be measured directly with a rheometer. We perform measurements on Earth for $0.01<\phi<0.05$; at these volume fractions, gels maintain their structural integrity under gravity over months. The DLCA gel time is relatively short, of the order of a few seconds; and in order to avoid heterogeneity in the networks, we reduce the salt concentration to $15 \mathrm{mM}$. We measure both storage and loss moduli, $G^{\prime}$ and $G^{\prime \prime}$, with a strain-controlled rheometer in an oscillatory-shear experiment using a double-walled Couette geometry. Measurements at fixed frequency under increasing strain amplitude show a linear elastic response at low strains, with a yield strain of 0.3 . We measure the frequency-dependent $G^{\prime}$ and $G^{\prime \prime}$ for different $\phi$ using a fixed strain amplitude of $\gamma=5 \times 10^{-3}$, well within the linear regime. The measured values of $G^{\prime}$ are about an

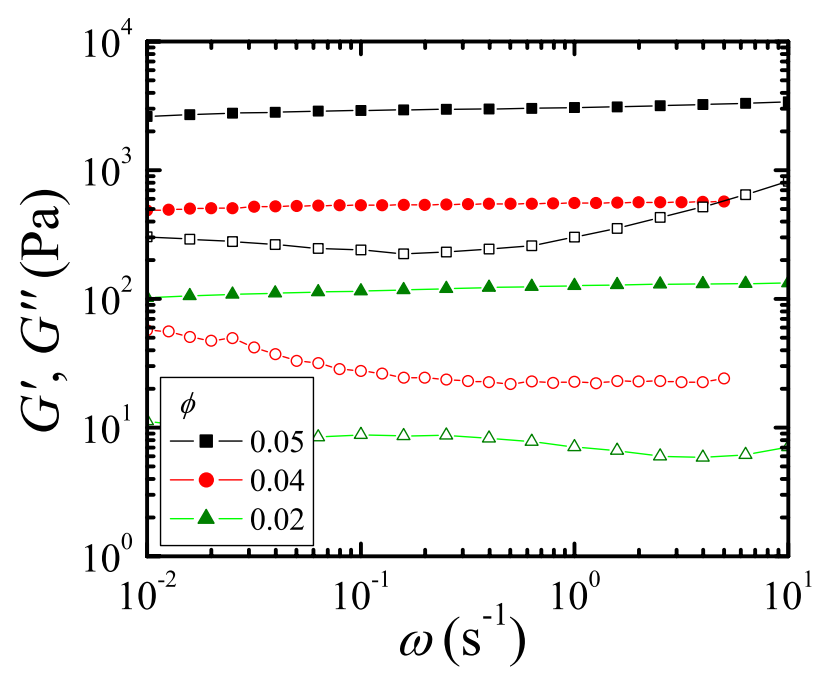

FIG. 2 (color online). Linear storage (filled symbols) and loss (open symbols) moduli for different volume fractions at strain amplitude $\gamma=5 \times 10^{-3}$. 
order of magnitude larger than $G^{\prime \prime}$ for all $\phi$ as shown in Fig. 2, demonstrating that these gels are indeed solid networks.

To determine the time evolution of the gels, we measure $G^{\prime}$ and $G^{\prime \prime}$ at fixed strain amplitude $\gamma=5 \times 10^{-3}$ and frequency $\omega=1 \mathrm{rad} / \mathrm{s}$ over a period of $12 \mathrm{~h}$, beginning upon gelation, as determined by the dominance of $G^{\prime}$ over $G^{\prime \prime}$. The samples all become stiffer with time, consistent with the development of a plateau in the DLS in the ISS experiment. At these higher $\phi$, the time evolution can be more precisely determined; for each of the samples, we find a power-law growth, $G^{\prime} \sim t^{0.4}$, over the entire measurement time, as shown in Fig. 3. To ascertain that the oscillatory strain does not influence the aging, we measure the modulus of the sample with $\phi=0.02$ only once every 30 min, allowing the sample to remain quiescent between measurements. The time dependence is unchanged, confirming that the strains applied do not affect $G^{\prime}$.

The elasticity of fractal colloidal gels is strongly $\phi$ dependent. However, the time dependence of $G^{\prime}$ is independent of $\phi$, and the data can all be scaled together; thus, the $\phi$ dependence of $G^{\prime}$ is directly reflected by the scale factor $\beta$. The dependence $\beta^{-1} \sim \phi^{3.6}$, shown in Fig. 3 (inset) is remarkably similar to that expected for fractal gels, where the macroscopic modulus is governed by the stiffness of the aggregate network. The elasticity of a fractal aggregate of size $R_{c}$ is determined by the spring constant $\kappa\left(R_{c}\right)=\kappa_{0} a^{2} / N R_{c}^{2}$ of its backbone, where $\kappa_{0}$ is the interparticle spring constant and $N$ is the number of particles in the chain $[15,18]$. This expression can be understood in an intuitive way, if one assumes that the primary contributions to elasticity are from bond bending. For a bent chain, a change in the length of the chain $\Delta l$ is translated into a change in the average local bending angle $\langle\Delta \theta\rangle$, resulting in an elastic energy of $U=\frac{1}{2} \kappa_{0}(a\langle\Delta \theta\rangle)^{2} N$.

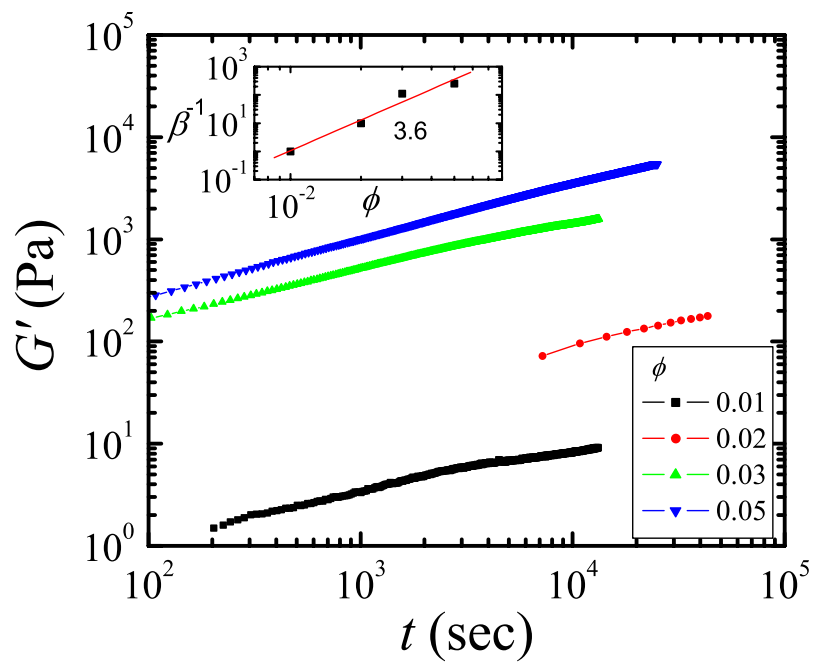

FIG. 3 (color online). Growth of the linear storage modulus $G^{\prime}$ with time for different volume fractions. Scale factors $\beta$ used to shift $G^{\prime}$ onto a single master curve (inset).
The total change in angles is $N \Delta \theta=\frac{\Delta l}{R_{c}}$, which is shared among all of the $N=R^{d_{b}}$ particles in the chain, where $d_{b}$ is the bond dimension. The spring constant therefore depends inversely on $R_{c}^{2}$, and inversely on $N$. The elastic modulus of the gel is then $G^{\prime}=\frac{\kappa\left(R_{c}\right)}{R_{c}}=\frac{\kappa_{0}}{a} \phi^{\left(3+d_{b}\right) /\left(3-d_{f}\right)}$; using $d_{b}=1.1$ as found in simulations [19], and $d_{f}=1.9$, we obtain an exponent of 3.7, in excellent agreement with our data.

The weakest link determines $\kappa_{0}$; we assume that this is the contact region between particles, which is a neck of size $r_{0}$, whose elastic properties are the same as that of the particles, as shown schematically in Fig. 4. The strain, $\gamma$, imposed by the bending of the necks, will be concentrated in a region of size $r_{0}^{3}$, resulting in a strain energy of $E \gamma^{2} r_{0}^{3}$, where $E$ is the Young's modulus of silica. A change in bond angle of $\Delta \theta$ will result in a change in elastic energy of $\kappa_{0}(a \Delta \theta)^{2}$; thus, the local spring constant is $\kappa_{0}=E r_{0}$. This allows us to relate the properties of the local bonds to the macroscopic modulus, and to deduce how changes in local bonding result in changes in network elasticity. At the microscopic level, either an increase in contact radius, or an increase in elasticity of the material in the contact region will lead to an increase in $\kappa_{0}$.

We assume that upon gelation particles form contacts at the molecular level. Particles in contact gain surface energy by deforming elastically, thereby increasing their contact area as described by the Johnson-Kendall-Roberts (JKR) theory [20]. The radius of the contact area reaches a maximum value, $r_{m}$, determined by a balance between elastic deformation and surface energies: $r_{m} \approx$ $\left(9 \sigma a^{2} / 4 E\right)^{1 / 3}$, where $\sigma$ is the surface tension. Substituting typical values for silica of $\sigma \approx 100 \mathrm{~mJ} / \mathrm{m}^{2}$ and $E \approx 10^{10} \mathrm{~Pa}$, we obtain $r_{m} \approx 1.3 \mathrm{~nm}$. Using the expression for the modulus, we calculate $G^{\prime} \approx 2 \times 10^{4} \mathrm{~Pa}$, for a gel at $\phi=0.05$. This is approximately twice the measured $G^{\prime}$ after $24 \mathrm{~h}$, indicating that the JKR contact may be still developing, consistent with the observation that $G^{\prime}$ continues to increase during the entire measurement time.

The time dependence of $G^{\prime}$ is $\phi$ independent, and SLS indicates that there is no change in the gel structure. This

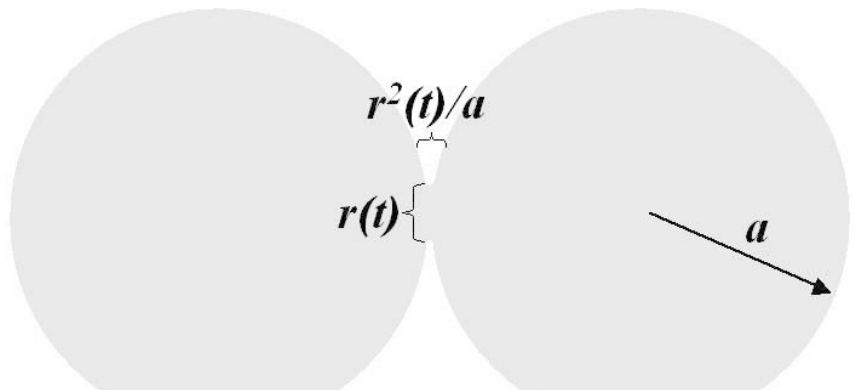

FIG. 4. Schematic of two sintering spheres. 
suggests that it is a time-dependent interparticle spring constant $\kappa_{0}(t)$ and not structural changes, that is responsible for increases in $G^{\prime}$. We assume that this time dependence arises from an increase in the size of the neck. One possibility is that this growth occurs by mass transfer; however, this is too slow to explain the observed growth in $G^{\prime}$. Instead, we hypothesize that the kinetics of bond formation is reaction limited, as formation of $\mathrm{Si}-\mathrm{O}-\mathrm{Si}$ bonds requires hydration of cations at the surface of the particles. Support for this comes from atomic force microscopy studies [21], which attribute a short-ranged repulsion to the presence of a counterion layer. We can use the measurements of $G^{\prime}(t)$ to estimate the bond activation energy, and the evolution of the contact area. The number of bonds required to make a JKR contact is $N_{m} \sim r_{m}^{2}$, and we estimate that the time scale for a bond to form is $\tau \approx$ $A_{f}{ }^{-1} e^{-\Delta U / k_{B} T}$, where $A_{f} \approx 10^{-13} \mathrm{sec}^{-1}$ is a molecular attempt frequency. The rate of formation of new bonds is $\frac{d N}{d t} \approx\left(N_{m}-N\right) / \tau \approx N_{m} / \tau$ for $N \ll N_{m}$. Since $r \sim \sqrt{N}$, its time evolution is $r \approx r_{0} \sqrt{t / \tau}$, where $r_{0}$ is the contact radius upon gelation. Substituting $r$ into our expressions for $\kappa_{0}$ and $G^{\prime}$, we obtain $G^{\prime} \sim t^{0.5}$, in reasonable agreement with our measurement, $G^{\prime} \sim t^{0.4}$. The time dependence requires $\Delta U \approx 33 k_{B} T$; this is comparable to known hydration enthalpies [21], supporting our hypothesis.

An alternative microscopic picture for the strengthening of the gel network is an increase in the elasticity of the bonded region. Hydrated silica surfaces are swollen relative to dry ones [22]; this could be due to a gel-like surface layer, composed of "polymeric" chains of $-\mathrm{Si}(\mathrm{OH})_{2}-\mathrm{O}-\mathrm{Si}(\mathrm{OH})_{2}-\mathrm{OH}$ groups. Measurements made in humid air indicate that bond strength also increases with contact time [22], purportedly as a result of consolidation of the gel layer. It is conceivable that, in water, this would lead to an increase in $E$, and therefore $\kappa_{0}(t)$; however, the nature of these effects is unclear, and difficult to quantify.

Our measurements show that changes in bonding at the particle level can significantly affect the macroscopic elasticity of colloidal gels. While the focus here is on silica gels, we expect similar behavior for gels of different materials. This is in sharp contrast with the more usual time evolution of elasticity due to changes in the network structure; this provides an alternate potential means for changing the time-dependent materials properties of colloidal gels.
This work was supported by NASA (NAG3-2284).

*Present address: GDPC cc 26, Universite Montpellier II, P. E. Bataillon, Montpellier 34000, France.

†Present address: Scitech Instruments, Inc., North Vancouver, BC, Canada V7J 2S5.

${ }^{\ddagger}$ Present address: Dept. of Physics, University of Konstanz, Konstanz, Germany.

${ }^{\S}$ Present address: Dept. of Chemical Engineering, UCSB, Santa Barbara, CA 93106, USA.

"Present address: NASA Marshall SpaceFlight Center, Huntsville, AL 35812, USA.

[1] B. Rinn, P. Maass, and J.-P. Bouchaud, Phys. Rev. B 64, 104417 (2001).

[2] P. G. Debenedetti and F. H. Stillinger, Nature (London) 410, 259 (2001).

[3] B. V. Derjaguin and L. Landau, Acta Physicochim URSS 14, 633 (1941).

[4] E. J. W. Verwey and J. T. G. Overbeek, Theory of Stability of Lyophilic Colloids (Elsevier, Amsterdam, 1948).

[5] S. Asakura and F. Oosawa, J. Chem. Phys. 22, 1255 (1954).

[6] W. Y. Shih, W.-H. Shih, and I. A. Aksay, J. Am. Ceram. Soc. 82, 616 (1999).

[7] V. Prasad et al., Faraday Discuss. 123, 1 (2003).

[8] H. M. Wyss et al., J. Colloid Interface Sci. 241, 89 (2001).

[9] L. Cipelletti et al., Phys. Rev. Lett. 84, 2275 (2000).

[10] C. T. Lant et al., Appl. Opt. 36, 7501 (1997).

[11] M.P. Doherty et al., NASA Technical Report No. NASA/ TM-2002-211371 (2002).

[12] S. Manley et al., Phys. Rev. Lett. 93, 108302 (2004).

[13] M. Carpineti et al., Phys. Rev. A 42, 7347 (1990).

[14] M. Carpineti and M. Giglio, Phys. Rev. Lett. 68, 3327 (1992).

[15] T. Gisler, R. C. Ball, and D. A. Weitz, Phys. Rev. Lett. 82, 1064 (1999).

[16] M. Carpineti and M. Giglio, Phys. Rev. Lett. 70, 3828 (1993).

[17] A. H. Krall and D. A. Weitz, Phys. Rev. Lett. 80, 778 (1998).

[18] Y. Kantor and I. Webman, Phys. Rev. Lett. 52, 1891 (1984).

[19] P. Meakin et al., J. Phys. A 17, L975 (1984).

[20] K. Johnson, K. Kendall, and A. Roberts, Proc. R. Soc. A 324, 301 (1971).

[21] I. U. Vakarelski, K. Ishimura, and K. Higashitmi, J. Colloid Interface Sci. 227, 111 (2000).

[22] G. Vigil et al., J. Colloid Interface Sci. 165, 367 (1994). 